Urologe 2019·58:615-616

https://doi.org/10.1007/s00120-019-0946-x

Online publiziert:27. Mai 2019

๑ Springer Medizin Verlag GmbH, ein Teil von Springer Nature 2019

\author{
J. Kranz ${ }^{1,2} \cdot$ J. Steffens ${ }^{1} \cdot$ A. Haferkamp ${ }^{3}$ \\ ${ }^{\prime}$ Klinik für Urologie und Kinderurologie, St.-Antonius-Hospital Eschweiler, Akademisches \\ Lehrkrankenhaus der RWTH Aachen, Eschweiler, Deutschland \\ ${ }^{2}$ Universitätsklinik und Poliklinik für Urologie, Universitätsklinikum Halle (Saale), Halle (Saale), \\ Deutschland \\ ${ }^{3}$ Urologische Klinik und Poliklinik, Universitätsklinikum der Johannes Gutenberg Universität, Mainz, \\ Deutschland
}

\title{
Funktionell-rekonstruktive Urologie
}

Angeborene und erworbene Funktionsstörungen des oberen und unteren Harntraktes stellen eine therapeutische Herausforderung dar.

Eine gezielte symptomorientierte sonographische, radiologische, urodynamische und neurourologische Diagnostik bilden die Basis für eine individualisierte Behandlung. Nur durch die Zusammenschau und Bewertung anatomischer und funktioneller Befunde kann eine konservative oder operative Verbesserung des beeinträchtigten urogenitalen Funktionszustandes erzielt werden.

Deshalb ist sowohl der gezielte Einsatz einer invasiven Diagnostik als auch das Angebot einer maßgeschneiderten chirurgischen Rekonstruktion wesentlich für den Behandlungserfolg. Vor diesem Hintergrund wurde das vorliegende Heft konzipiert. Es werden bewährte Verfahren der operativen Behandlung des weiblichen Urogenitalprolapses vorgestellt (Neymeyer et al.). Aufgrund einer immer noch zu häufigen und unkritischen Implantation von Bändern wird die exakte Indikationsstellung zur operativen Behandlung der weiblichen Belastungsharninkontinenz dargestellt (Hampel). Nach Ausschöpfung konservativer Behandlungsmöglichkeiten kann eine sakrale Neuromodulation eine effektive Behandlungsoption bei der therapierefraktären überaktiven Blase, nicht-obstruktiver Harnretention und fäkaler Inkontinenz sein (Girtner et al.).

Gegenüber der weiblichen Harninkontinenz, die zu einer der zehn wichtigsten Diagnosen bei erwachsenen Frauen zählt, stellt die männliche
Harninkontinenz eine Minderheit dar und ist meist iatrogene Folge von Prostataeingriffen. Im Gegensatz zur Frau ist die Bildung eines spannungsfreien Widerlagers unter der Harnröhre kein operatives Behandlungsziel, da eine Hypermobilität der Harnröhre nicht die Ursache der Harninkontinenz ist. Die Operationsverfahren zielen beim Mann auf einen Ausgleich des Deszensus der hinteren Harnröhre (Elevation) oder passiven Druckausübung auf die Harnröhre (Kompression) mit Erhöhung des urethralen Widerstandes hin (Mühlstädt et al.). Die faszinierenden Rekonstruktionsmöglichkeiten meist iatrogener Harnleiterverletzungen werden abschließend eindrucksvoll dargestellt (Stühler et al.). Bei längerstreckigen Ureterdefekten kann durch Transposition körpereigenen Materials (Darm, Mundschleimhaut, Omentum majus) ein zufriedenstellendes Substitut mit guten funktionellen Ergebnissen hergestellt werden.

Dieses Themenheft verdeutlicht die Vielseitigkeit unseres Faches und mahnt zur Wissensaneignung nicht nur onkologischer Hauptthemen, sondern auch zur Beschäftigung mit diesem wesentlichen urologischen Teilgebiet.

Vor dem Hintergrund konkurrierender Nachbardisziplinen soll die Lektüre dieses Heftes auch ein Ansporn zur Beschäftigung mit Funktionsstörungen des Harntraktes sein. Denn nur die umfassende Bewertung anatomischer und funktioneller Aspekte in jedem Lebensalter wird unserem Fach die verdiente Anerkennung der chirurgischen Nachbarfächer sichern. 


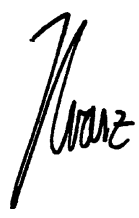

Hier steht eine Anzeige.

PD Dr. med. J. Kranz

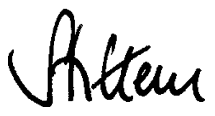

Prof. Dr. med. J. Steffens

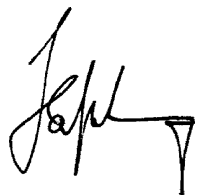

Prof. A. Haferkamp

\section{Korrespondenzadresse}

PD Dr. med. J. Kranz
Klinik für Urologie
und Kinderurologie,
St.-Antonius-Hospital
Eschweiler, Akademisches
Lehrkrankenhaus der RWTH
Aachen
Dechant-Deckers-Straße 8,
52249 Eschweiler,
Deutschland
jennifer.kranz@
sah-eschweiler.de

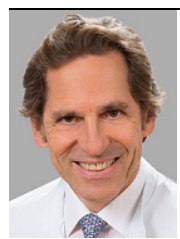

Prof. Dr. med. J. Steffens

Klinik für Urologie

und Kinderurologie,

St.-Antonius-Hospital

Eschweiler, Akademisches

Lehrkrankenhaus der RWTH

Aachen

Dechant-Deckers-Straße 8,

52249 Eschweiler,

Deutschland

Joachim.steffens@

sah-eschweiler.de

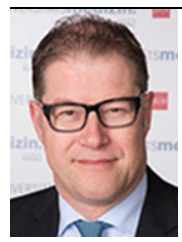

Univ.-Prof. Dr. med.

A. Haferkamp

Urologische Klinik und Poli-

klinik, Universitätsklinikum

der Johannes Gutenberg

Universität

Langenbeckstraße 1,

55131 Mainz, Deutschland

axel.haferkamp@unimedizinmainz.de

Interessenkonflikt. J. Kranz, J. Steffens und A. Haferkamp geben an, dass kein Interessenkonflikt besteht 